\title{
Derivados financieros y los efectos del fenómeno El Niño en la rentabilidad de las empresas pesqueras y agroindustriales peruanas
}

\author{
Financial derivatives and the effects of El Niño phenomenon on the profitability of \\ Peruvian fishing and agro-industrial companies
}

${ }^{1}$ Pedro Pablo Chambi Condori

Departamento Académico de Ingeniería Comercial. Universidad Nacional Jorge Basadre Grohmann. Tacna - Perú.

E - mail: pchambi@unjbg.edu.pe

\section{RESUMEN}

El objetivo del presente artículo fue demostrar el efecto del fenómeno El Niño (FEN) en la rentabilidad patrimonial de las empresas pesqueras y agroindustriales peruanas, y su cobertura con la adaptación de contratos de derivados. La investigación fue cuantitativa, descriptiva y explicativa, teniendo como base la cuantificación de factores que influyen en la disminución de la rentabilidad patrimonial de las empresas pesqueras y agroindustriales peruanas, en el periodo 2010 a 2018. La muestra seleccionada por convención estuvo conformada por 4 empresas que cotizan en la Bolsa de Valores de Lima. Por un lado, obteniendo los estados financieros anuales de las empresas y, por otro lado, el registro estadístico de la presencia del FEN y la temperatura de las regiones en las que están ubicadas las empresas en estudio. Teniendo, como resultado, evidencias de incidencia negativa por parte de los factores temperatura y del FEN en la rentabilidad patrimonial de las empresas, cuyos valores se exhiben en la tabla 2 y modelo (1) y el precio de la cobertura con derivados. Los resultados obtenidos muestran el efecto negativo de los factores temperatura y del FEN en la rentabilidad patrimonial de las empresas pesqueras y agroindustriales peruanas, cuyo aseguramiento es posible adoptando la contratación en OTC de opciones de venta en posición larga, que asegure la pérdida de valor en la rentabilidad de las empresas.

Palabras clave: Derivados, rentabilidad, riesgos climáticos.

\section{ABSTRACT}

The objective of this article was to demonstrate the effect of El Niño phenomenon (FEN) on the equity profitability of Peruvian fishing and agro-industrial companies and their coverage with the adaptation of derivative contracts. The research was quantitative, descriptive and explanatory based on the quantification of factors that influence the decrease in the profitability of the Peruvian fishing and agro-industrial companies in the period of 2010 to 2018. The sample selected by convention was made up of 4 companies that are listed on the Lima Stock Exchange, on the one hand, obtaining the annual financial statements of the companies and, on the other hand, the statistical record of the presence of the FEN and the temperature of the regions in which the studied companies are located. Obtaining as evidence of negative incidence by the Temperature and FEN factors on the equity profitability of the companies, whose values are shown in Table 2 and model (1) and the price of hedging with derivatives. The results obtained show the negative effect of temperature and FEN factors on the profitability of Peruvian fishing and agro-industrial companies, whose insurance is possible by adopting the OTC contracting of long-term options, which ensures the loss of value in the profitability of companies.

Keywords: Derivatives, profitability, weather risks. 


\section{INTRODUCCIÓN}

Los eventos a consecuencia del fenómeno El Niño (FEN), que ha vivido el Perú en los últimos 40 años, han sido ampliamente estudiadas por académicos extranjeros y nacionales, así Galarza y Kamiche (2012), en estudio de evaluación de impactos del FEN en la economía regional de Piura, Lambayeque y Libertad, norte del Perú, abordan los fenómenos ocurridos durante los años 1982-1983 y 1997-1998. En dicho estudio, llegan a la conclusión que el $80 \%$ de los daños físicos han tenido impacto en la agricultura y la pesca en la zona norte del Perú, y el $20 \%$ en la zona sur. El $42 \%$ de los daños, los ocasionaron las excesivas lluvias y el resto (58 \%), la sequía.

Olivares y Zingaretti (2017), en un estudio realizado sobre los efectos de cambio climático en la agricultura de Venezuela en el periodo 1980 a 2014, utilizando la metodología estadística basada en el análisis multivariado, permitió delinear grupos de años que mostraron patrones semejantes del índice de sequía meteorológica en función a la serie temporal. El estudio constituye la columna vertebral para la planificación del espacio y el fortalecimiento de las estrategias para la seguridad alimentaria en esas localidades.

Para Gutiérrez y Sueiros (2015):

El primer problema transzonal se refiere a la contaminación antrópica del Gran Ecosistema Marino de la Corriente de Humboldt (GEMCH), la cual resulta del asentamiento de la mayor proporción humana e industrial en zonas costeras, con consecuencias económicas y ambientales. El segundo alude al uso no óptimo de los recursos pesqueros, o sea, en algunos casos se observa una declinación de la abundancia o de capturas de algunas especies que son claves para la seguridad alimentaria. Pero este problema también se refiere a la baja calidad de la producción pesquera, o a deficiencias en su condición sanitaria, lo que conlleva impactos económicos, sociales y ambientales. El tercer problema, de tipo común, concierne a la elevada pesca ilegal o pesca incidental y descartes que, se ha reconocido, ocurren en ambos países, Perú y Chile. (p.9)

Martelo (1998) explica así el fenómeno de El Niño:

El Niño es uno de los varios ciclos climáticos irregulares que se producen por la interacción entre el océano y la atmósfera. Es un fenómeno propio de la variabilidad climática (no del cambio climático), que se manifiesta mediante cambios en los patrones normales de comportamiento de:

las corrientes oceánicas,

los flujos de viento, $\mathrm{y}$

la distribución de la precipitación y la temperatura.

El Niño no es un fenómeno nuevo; existen pruebas de que ha existido durante miles de años. Sin embargo, no fue sino hasta el último decenio en que se logró comprender satisfactoriamente cómo se forma y se mantiene.

En los últimos años, hemos aprendido que el calentamiento del mar en diciembre frente a las costas de Perú y Ecuador es sólo una parte de un fenómeno mucho más amplio, que involucra a todo el océano Pacífico tropical y que también tiene manifestaciones atmosféricas, que se observan como cambios en la distribución normal de la precipitación. Los cambios en el sistema atmosférico se denominan "Oscilación del Sur", por lo que los científicos crearon el acrónimo ENOS (E1 NiñoOscilación del Sur) para referirse al fenómeno combinado de cambios oceánicos y atmosféricos. (p. 48-55)

¿Cómo impacta sobre la naturaleza?

- Sequías al este de Australia e Indonesia, que se extienden hasta las Filipinas.

- Aumento de las precipitaciones en la costa pacífica de América, especialmente en Perú y Ecuador.

- Incremento de la incidencia de tormentas tropicales y de huracanes en el Pacífico.

- Tendencia a inviernos astronómicos en el hemisferio norte (diciembre) más cálidos de lo normal en la costa pacífica de Estados Unidos y Alaska.

¿Y sus secuelas en otras partes del mundo?

- Sequías en el Sahel (la zona al sur del desierto del Sahara). 
- Sequías en el Nordeste de Brasil (temporada lluviosa de febrero a mayo).

- Disminución de las lluvias provocadas por los vientos monzones de verano (julio-septiembre) sobre India.

- Disminución de las tormentas tropicales y de los huracanes en el Atlántico.

- Aumento de las precipitaciones en la zona del Golfo de México, el sur de Brasil, Uruguay, Paraguay y el norte y centro de Argentina.

- Disminución de las precipitaciones en el sureste de Venezuela.

El mundo científico está alarmado porque la ocurrencia de estos fenómenos, en el hemisferio norte, se realizaba en mayo. Ahora, son imprevisibles y acontecen en cualquier época del año.

El tema es importante porque la imagen institucional del Perú, como país exportador de harina de pescado, está en juego y los 150000 empleos que dependen de esta actividad (Sociedad Nacional de Pesquería, 2011).

El contenido es de interés porque un sector industrial sin materia prima a procesar afecta la economía de las empresas, disminuye el nivel de impuestos al Estado y deja sin trabajo a sus colaboradores, disminuyendo el nivel de vida en el sector y elevando la pobreza.

La cuestión no es novedad, pero ha ganado notoriedad, porque su efecto multiplicador, afecta a miles de personas tanto en el sector nacional como internacional.

Aguirre y Méndez(2015), desde Trujillo, norte del Perú, explican:

Para la presente investigación económica se aplicará el diseño de series de tiempo, que permitirá determinar el modelo que explique la relación del PBI pesquero y PBI nacional. El diseño comprende el Método de Mínimos Cuadrados para la obtención del modelo que explique el comportamiento de las variables y series, facilitando los propósitos correspondientes. (p.29)

Además, concluyen:

(1). En la presente investigación se ha determinado que durante el periodo 1970-2014 el sector pesquero es una variable que presenta un impacto pequeño en el PBI real, esto se confirma mediante la evidencia empírica del modelo econométrico, que al aumentar el sector pesquero en $1 \%$ el crecimiento de la economía (PBI real) crece en 0.04\%. También, se corrobora con el peso o participación que tiene el sector pesquero de menos de 1\% en el PBI real. (p. 68-69)

Por otro lado, Caparrós (2006) conceptúa al riesgo climático como el riesgo de incurrir en pérdidas no esperadas, como consecuencia de movimientos adversos en la temperatura debido a la presencia de lluvias, sequías y heladas. Ya no es posible evitar el cambio climático, pero sí es posible proteger en cierta medida las economías y sociedades contra su impacto. A principios de los 90, se crearon los primeros derivados de clima (OTC); y, a finales de dicha década, se empezaron a negociar en la Bolsa de Chicago derivados del clima estandarizados, para poder realizar operaciones de cobertura a todas aquellas empresas que se encuentran en sectores que sufren el riesgo climático.

Castro (2009) afirma que los derivados climáticos constituyen una herramienta para reducir el riesgo financiero que enfrentan empresas en donde sus ganancias están correlacionados a las condiciones climáticas. Los derivados proporcionan coberturas frente a eventos altamente probables como las lluvias, sequías y variabilidad alta en la temperatura. Un derivado del clima se basa en un subyacente que no es negociable en el mercado de activos financieros. En el caso de una opción climática aplicada a la pesca y la agricultura, el objetivo es cubrir la incertidumbre sobre volúmenes producidos. La primera transacción OTC sobre derivados del clima se realizó en 1996, cuando Koch Industries y ENRON completaron un swap para el invierno de 1997 en Milwaukee, Wisconsin.

Las primeras aplicaciones de derivados fueron realizadas en los Estados Unidos en 1997, bajo el tipo de contratos OTC (over the counter), tipo de contrato entre dos empresas privadas fuera del mercado organizado. El primer contrato que aparece registrado en la literatura tuvo lugar entre Enron Capital y Trade Resources, en el ámbito del sector energético, contrato que fue diseñado por Koch Industries y fue concebido como un swap sobre la variable temperatura para el invierno del año 1997 en la ciudad de Milwaukee de Estados Unidos. El contrato en referencia tuvo como antecedente al contrato entre Aquila Energy y Consolidated Edison Co. En julio de 1996, en la compra y venta de electricidad (Hull, 2002). 
El tipo de contrato OTC se utiliza para denominar a todas aquellas operaciones que se negocian fuera de un mercado organizado. Estos mercados tienen características que los hacen muy atractivos para los inversionistas y algunas de ellas son: los contratos son preparados a la medida sobre diversos subyacentes, no hay pagos a intermediarios, se liquidan en su fecha de vencimiento, el riesgo de incumplimiento es asumido por los contratantes ante un juez competente clausulado en el contrato, no está sujeta a regulación (Soriano, 2012).

Benito (2012) afirma que en los contratos de opciones se encuentra el tipo de contrato OTC. La ventaja de dicho contrato permite que una institución financiera puede adaptarlas para atender las necesidades específicas de un tesorero corporativo o administrador de fondos. Los factores que influyen en el valor de una opción sobre acciones. Hay 6 factores que influyen en el valor de una opción sobre acciones:

- El precio actual del subyacente

- El precio de ejercicio (suma asegurada).

- La fecha de vencimiento del contrato.

- La volatilidad del subyacente

- La tasa libre de riesgo (p.16).

Tabla 1. Impacto del Fenómeno El Niño en el Perú

\begin{tabular}{|c|c|}
\hline Actividad o sector afectado & Efectos del fenómeno El Niño (FEN) \\
\hline Pesca & $\begin{array}{l}\text { - Principal actividad económica afectada } \\
\text { - Migración de las especies marinas a áreas de menor temperatura. } \\
\text { - Afectación de la pesca industrial por escasez de anchoveta. } \\
\text { - Caída de la producción de harina de pescado. }\end{array}$ \\
\hline & $\begin{array}{l}\text { - Afectada por el incremento de la temperatura y humedad así como por } \\
\text { las inundaciones. }\end{array}$ \\
\hline Agricultura & - Reducción de las cosechas de tubérculos y frutas \\
\hline Exportaciones & - Reducción de exportaciones pesqueras y agrícolas. \\
\hline Energía & - Daños en las centrales hidroeléctricas \\
\hline Transporte & - Destrucción de carreteras y puentes. \\
\hline
\end{tabular}

Fuente: Adaptado de MINAM Perú, 2016.

En la tabla 1 se observa de manera clara los efectos del FEN en diversos sectores de la economía peruana a 2016.

\section{Objetivo:}

El objetivo general de la investigación es determinar y explicar el efecto de cambios de temperatura en la rentabilidad de las empresas pesqueras y agroindustriales que cotizan en el mercado de valores del Perú y la cobertura de riesgos climáticos bajo la teoría de opciones.

\section{Hipótesis:}

Existen evidencias del efecto de cambios de temperatura en la rentabilidad de las empresas pesqueras y agroindustriales que cotizan en el mercado de valores del Perú y la cobertura de riesgos climáticos bajo la teoría de opciones.

\section{MÉTODO Y MATERIALES}

Tipo de investigación cuantitativa, explicativa y longitudinal. Se selecciona por convención a 2 empresas pesqueras y 2 empresas agroindustriales que cotizan en la Bolsa de Valores de Lima, en el periodo de 2000 a 2018. Se recoge la información de estados financieros, publicada en la base de datos de la Bolsa de Valores de 
Lima y los cambios de temperatura y la presencia del fenómeno de El Niño a través de la base de datos que administra SENAMHI Perú.

Se organizan la serie de tiempo acerca del ROE de las empresas y, por otro lado, la serie de datos de la temperatura. Se obtienen la variación de la temperatura en el tiempo y la variación del ROE de las empresas. Se procesan los datos utilizando el panel data, para obtener el modelo causal que describen y muestran los efectos del cambio de temperatura sobre la rentabilidad de las empresas pesqueras y agroindustriales peruanas. Para, finalmente, utilizar el modelo de cobertura de riesgos con opciones PUT en posición larga, buscando modelar el aseguramiento de la rentabilidad de las empresas.

Para estudiar los efectos de fenómeno El Niño (FEN) en las actividades pesqueras y agrícolas en el Perú, por convención, se han seleccionado dos empresas pesqueras y dos empresas agroindustriales, cuyas acciones se cotizan en la Bolsa de Valores de Lima, en el periodo de 2010 a 2018. Los efectos se ven reflejados en los resultados de las empresas, precisamente, en la rentabilidad patrimonial. Se formula el modelo del ROE explicado por la temperatura, la presencia o no del FEN en un determinado año y del comportamiento de la economía, cuya expresión matemática se expresa de la forma siguiente:

$R O E=f\left(\right.$ Temperatura, $\left.E l_{N i n ̃ o}, P B I\right)$ y la expresión en panel data es el siguiente:

$R O E_{i t}=\beta_{0}+\beta_{i t}(T)+\beta_{i t}(F E N)+\beta_{i t}(P B I)+\varepsilon$, en donde

ROE : $\quad$ Rentabilidad patrimonial de la empresa.

T : : Temperatura

FEN : $\quad$ variable dicotómica que toma valores 1: presencia de FEN, 0: no FEN.

PBI : $\quad$ El comportamiento de la economía regional peruana en donde se ubican cada una de las empresas estudiadas.

Una vez identificado el impacto de FEN en las actividades agrícolas y pesqueras, se busca modelar la protección de los riesgos climáticos con opciones financieras y, como instrumento de aseguramiento, la posición larga de PUT para conservar el valor de la rentabilidad de las empresas afectadas. Para lo cual, se consultan los modelos de valoración de opciones financieras de Black \& Scholes (Hull, 2002, p.273), aplicable a opciones que se ejercitan en su fecha de vencimiento.

$$
\begin{gathered}
c=X * N(d 1)-E *\left(e^{-R_{f} * T}\right) * N\left(d_{2}\right) \\
p=X *\left[N\left(d_{1}\right)-1\right]-E *\left(e^{-R_{f} * T}\right) *\left[N\left(d_{2}\right)-1\right]
\end{gathered}
$$

En donde:

c : $\quad$ Valor de la opción CALL

$\mathrm{p} \quad$ : $\quad$ Valor de la opción PUT

$\begin{array}{lll}d_{1} & = & \frac{\ln \left(\frac{\mathrm{X}}{\mathrm{E}}\right)+\mathrm{R}_{\mathrm{f}} * \mathrm{~T}+\frac{1}{2}\left(\sigma^{2}\right) * \mathrm{~T}}{\sigma \sqrt{\mathrm{T}}} \\ d_{2} & = & \mathrm{d}_{1}-\sigma \sqrt{\mathrm{T}}\end{array}$

$N\left(d_{1}\right)=$ distr.norm. estand $\left(d_{1}\right)$

$N\left(d_{2}\right)=$ distr.norm.estand $\left(d_{2}\right)$

$\mathrm{N}(\mathrm{d}) \quad$ : es la función de distribución de probabilidad para una variable normal estandarizada.

E : : Es el precio de ejercicio de la opción, para el caso la tasa de aseguramiento.

Rf : $\quad$ Tasa libre de riesgo

$\mathrm{T}$ : : Tiempo de vigencia del contrato

e : : 2.718281, valor constante.

$\mathrm{X} \quad$ : $\quad$ Valor del subyacente, que en el caso nuestro es $R O E=\frac{\text { Utilidad neta }}{\text { Patrimonio }}$ 
Una vez obtenido el valor de aseguramiento, se hace necesario evaluar la sensibilidad que describe el subyacente, para esto se utiliza la función delta put.

$\left.\frac{d p}{d X}=N\left[\left(d_{1}\right)-1\right)\right]$

\section{RESULTADOS}

A través de los estados financieros se constata que en el periodo analizado, las empresas pesqueras han sido las más afectadas, obteniendo resultados económicos negativos, por la disminución sustancial en ventas hasta en - 25 $\%$ anual, e incurriendo en mayores costos financieros. Las empresas agroindustriales han sido afectadas en menor medida, en promedio en el orden de $-12 \%$ anual en los periodos de presencia del fenómeno de El Niño.

Haciéndose necesaria la presencia de instrumentos de aseguramiento, tal que conserve la rentabilidad planeada, situación que es abordada en el estudio mediante la posición larga de PUT, bajo el tipo de contrato OTC.

Tal como se puede apreciar en la tabla 2, en el año 2016, la pesquera Exalmar obtiene rentabilidad de -11 \%, Agroindustrial Paramonga el 2.7 \%. Es así que, en el periodo de la presencia del FEN (2015, 2016 y 2017), las empresas han mostrado rentabilidad patrimonial disminuida como consecuencia del efecto del FEN. Por otro lado, en los años en los que no se ha manifestado la presencia del FEN, las empresas han mostrado ganancias aceptables, así ocurre con las empresas Exalmar y Austral Group en 2011 con ganancias bastante aceptables, superiores al $7 \%$ anual. En los periodos de la presencia del FEN, se constata la temperatura en rangos superiores a $30^{\circ} \mathrm{C}$. En dichas regiones, bajo condiciones normales, estuvieron alrededor de $20^{\circ} \mathrm{C}$.

Tabla 2. Factores Incidentes en el ROE

\begin{tabular}{|c|c|c|c|c|}
\hline \multirow[b]{2}{*}{ EMPRESA } & \multicolumn{4}{|c|}{ TEMPERATU FENÓMEN } \\
\hline & ROE & RA MÁXIMA & O_NIÑO & C.PBI \\
\hline 2010 PESQUERA EXALMAR SAA & $7.89 \%$ & 30.3 & 1 & 6.8 \\
\hline 2011 PESQUERA EXALMAR SAA & $9.92 \%$ & 30.8 & 0 & 5.8 \\
\hline 2012 PESQUERA EXALMAR SAA & $9.18 \%$ & 31.0 & 1 & 9.5 \\
\hline 2013 PESQUERA EXALMAR SAA & $0.31 \%$ & 30.0 & 0 & 3.6 \\
\hline 2014 PESQUERA EXALMAR SAA & $6.05 \%$ & 31.3 & 0 & 2.1 \\
\hline 2015 PESQUERA EXALMAR SAA & $2.20 \%$ & 31.7 & 1 & 4.4 \\
\hline 2016 PESQUERA EXALMAR SAA & $-11.00 \%$ & 32.3 & 1 & 2.5 \\
\hline 2017 PESQUERA EXALMAR SAA & $3.00 \%$ & 30.6 & 1 & 2.6 \\
\hline 2018 PESQUERA EXALMAR SAA & $12.00 \%$ & 31.3 & 0 & 4.1 \\
\hline 2010 AUSTRAL GROUP SAA & $7.67 \%$ & 21.3 & 1 & 5.8 \\
\hline 2011 AUSTRAL GROUP SAA & $14.79 \%$ & 21.6 & 0 & 4.5 \\
\hline 2012 AUSTRAL GROUP SAA & $10.74 \%$ & 22.3 & 1 & 7.7 \\
\hline 2013 AUSTRAL GROUP SAA & $1.05 \%$ & 21.3 & 0 & 4.4 \\
\hline 2014 AUSTRAL GROUP SAA & $-5.23 \%$ & 22.1 & 0 & 1.5 \\
\hline 2015 AUSTRAL GROUP SAA & $-1.51 \%$ & 23.4 & 1 & 2.3 \\
\hline 2016 AUSTRAL GROUP SAA & $-5.14 \%$ & 23.3 & 1 & 0.9 \\
\hline 2017 AUSTRAL GROUP SAA & $-4.41 \%$ & 23.1 & 1 & 1.7 \\
\hline 2018 AUSTRAL GROUP SAA & $17.88 \%$ & 22.4 & 0 & 5.3 \\
\hline 2010 AGROINDUSTRIAL LAREDO SAA & $9.81 \%$ & 24.5 & 1 & 5.8 \\
\hline 2011 AGROINDUSTRIAL LAREDO SAA & $16.59 \%$ & 25.1 & 0 & 4.5 \\
\hline 2012 AGROINDUSTRIAL LAREDO SAA & $10.15 \%$ & 26.5 & 1 & 7.7 \\
\hline 2013 AGROINDUSTRIAL LAREDO SAA & $6.53 \%$ & 24.7 & 0 & 4.4 \\
\hline 2014 AGROINDUSTRIAL LAREDO SAA & $7.28 \%$ & 26.2 & 0 & 1.5 \\
\hline 2015 AGROINDUSTRIAL LAREDO SAA & $1.01 \%$ & 27.1 & 1 & 2.3 \\
\hline 2016 AGROINDUSTRIAL LAREDO SAA & $6.22 \%$ & 27.0 & 1 & 0.9 \\
\hline 2017 AGROINDUSTRIAL LAREDO SAA & $3.20 \%$ & 26.3 & 1 & 1.7 \\
\hline 2018 AGROINDUSTRIAL LAREDO SAA & $0.22 \%$ & 25.3 & 0 & 5.3 \\
\hline 2010 AGROINDUSTRIAL PARAMONGA & $16.47 \%$ & 21.3 & 1 & 5.8 \\
\hline 2011 AGROINDUSTRIAL PARAMONGA & $15.59 \%$ & 21.6 & 0 & 4.5 \\
\hline 2012 AGROINDUSTRIAL PARAMONGA & $1.78 \%$ & 22.3 & 1 & 7.7 \\
\hline 2013 AGROINDUSTRIAL PARAMONGA & $-0.79 \%$ & 21.3 & 0 & 4.4 \\
\hline 2014 AGROINDUSTRIAL PARAMONGA & $-2.47 \%$ & 22.1 & 0 & 1.5 \\
\hline 2015 AGROINDUSTRIAL PARAMONGA & $2.10 \%$ & 23.4 & 1 & 2.3 \\
\hline 2016 AGROINDUSTRIAL PARAMONGA & $2.70 \%$ & 23.3 & 1 & 0.9 \\
\hline 2017 AGROINDUSTRIAL PARAMONGA & $6.51 \%$ & 23.1 & 1 & 1.7 \\
\hline
\end{tabular}


De los resultados presentados en la tabla 3, se constata la incidencia negativa del FEN y de la variable temperatura en la rentabilidad patrimonial de las empresas pesqueras y agroindustriales, en el periodo 2010 a 2018. La variable que tiene mayor incidencia es la presencia del FEN, seguido de la variable temperatura. Por otro lado, el comportamiento de la economía regional, en donde están ubicadas las empresas, incide positivamente en los resultados de las empresas.

Tabla 3. Modelo de panel data del ROE de las empresas

Dependent Variable: ROE

Method: Panel Least Squares

Date: 03/25/20 Time: 19:33

Sample: 20102018

Periods included: 9

Cross-sections included: 4

Total panel (balanced) observations: 36

\begin{tabular}{lrrrr}
\hline \multicolumn{1}{c}{ Variable } & Coefficient & Std. Error & t-Statistic & \multicolumn{1}{c}{ Prob. } \\
\hline C & 6.8788 & 22.2517 & 0.3091 & 0.7594 \\
PBI_REGION & 0.0449 & 0.2756 & 0.1628 & 0.8718 \\
TEMPERATURA & -0.2503 & 0.8656 & -0.2891 & 0.7745 \\
FNINO & -1.0952 & 1.2039 & -0.9097 & 0.3705 \\
& Effects Specification & & \\
Cross-section fixed (dummy variables) & & & \\
R-squared & 0.06030507 & Mean dependent var & 0.0982 \\
Adjusted R-squared & -0.13411457 & S.D. dependent var & 2.82195438 \\
S.E. of regression & 3.0052351 & Akaike info criterion & 5.21125445 \\
Sum squared resid & 261.911702 & Schwarz criterion & 5.51916091 \\
Log likelihood & -86.8025801 & Hannan-Quinn criter. & 5.31872205 \\
F-statistic & 0.31017994 & Durbin-Watson stat & 1.45702972 \\
Prob(F-statistic) & 0.92647634 & & \\
\hline
\end{tabular}

Fuente: Bolsa de Valores de Lima y BCRP.

En el modelo que se enuncia a continuación, se visualiza claramente la incidencia de las variables en la rentabilidad patrimonial de las empresas.

Modelo (1):

$$
R O E=6.8788+0.0449 * P B I_{R E G I O N}-0.2503 * \text { TEMPERATURA }-1.0952 * \text { FNIÑ } O+E
$$

Tabla 4. El precio y la sensibilidad del contrato

\begin{tabular}{|c|c|c|c|c|c|c|c|c|c|}
\hline & $E=$ & $12.00 \%$ & & & & & & & \\
\hline ROE & VOLATILIDAD & $\mathrm{Rf}$ & TIEMPO & $\mathrm{d} 1$ & $\mathrm{~d} 2$ & $\mathrm{~N}(\mathrm{~d} 1)$ & $\mathrm{N}(\mathrm{d} 2)$ & Delta Put & Put \\
\hline $0 \%$ & $40 \%$ & $8 \%$ & 0.25 & $\#_{j} \mathrm{NUM} !$ & $\# \mathrm{~N} \mathrm{NUM}$ & ${ }^{*} \mathrm{NUM} !$ & $\#_{j} \mathrm{NUM}$ & $\#$; NUM! & ${ }^{*} \mathrm{~N} \mathrm{NUM}$ \\
\hline $2 \%$ & $40 \%$ & $8 \%$ & 0.25 & -8.76 & .8 .96 & 0.00 & 0.00 & -1.00 & 0.0976 \\
\hline $4 \%$ & $40 \%$ & $8 \%$ & 0.25 & -5.29 & -5.49 & 0.00 & 0.00 & -1.00 & 0.0776 \\
\hline $6 \%$ & $40 \%$ & $8 \%$ & 0.25 & -3.27 & -3.47 & 0.00 & 0.00 & -1.00 & 0.0576 \\
\hline $8 \%$ & $40 \%$ & $8 \%$ & 0.25 & -1.83 & -2.03 & 0.03 & 0.02 & -0.97 & 0.0378 \\
\hline $10 \%$ & $40 \%$ & $8 \%$ & 0.25 & -0.71 & -0.91 & 0.24 & 0.18 & -0.76 & 0.0202 \\
\hline $12 \%$ & $40 \%$ & $8 \%$ & 0.25 & 0.20 & 0.00 & 0.58 & 0.50 & -0.42 & 0.0083 \\
\hline $14 \%$ & $40 \%$ & $8 \%$ & 0.25 & 0.97 & 0.77 & 0.83 & 0.78 & -0.17 & 0.0027 \\
\hline $16 \%$ & $40 \%$ & $8 \%$ & 0.25 & 1.64 & 1.44 & 0.95 & 0.92 & -0.05 & 0.0007 \\
\hline $18 \%$ & $40 \%$ & $8 \%$ & 0.25 & 2.23 & 2.03 & 0.99 & 0.98 & -0.01 & 0.0002 \\
\hline $20 \%$ & $40 \%$ & $8 \%$ & 0.25 & 2.75 & 2.55 & 1.00 & 0.99 & 0.00 & 0.0000 \\
\hline $22 \%$ & $40 \%$ & $8 \%$ & 0.25 & 3.23 & 3.03 & 1.00 & 1.00 & 0.00 & 0.0000 \\
\hline $24 \%$ & $40 \%$ & $8 \%$ & 0.25 & 3.67 & 3.47 & 1.00 & 1.00 & 0.00 & 0.0000 \\
\hline $26 \%$ & $40 \%$ & $8 \%$ & 0.25 & 4.07 & 3.87 & 1.00 & 1.00 & 0.00 & 0.0000 \\
\hline $28 \%$ & $40 \%$ & $8 \%$ & 0.25 & 4.44 & 4.24 & 1.00 & 1.00 & 0.00 & 0.0000 \\
\hline $30 \%$ & $40 \%$ & $8 \%$ & 0.25 & 4.78 & 4.58 & 1.00 & 1.00 & 0.00 & 0.0000 \\
\hline $32 \%$ & $40 \%$ & $8 \%$ & 0.25 & 5.10 & 4.90 & 1.00 & 1.00 & 0.00 & 0.0000 \\
\hline $34 \%$ & $40 \%$ & $8 \%$ & 0.25 & 5.41 & 5.21 & 1.00 & 1.00 & 0.00 & 0.0000 \\
\hline $36 \%$ & $40 \%$ & $8 \%$ & 0.25 & 5.69 & 5.49 & 1.00 & 1.00 & 0.00 & 0.0000 \\
\hline $38 \%$ & $40 \%$ & $8 \%$ & 0.25 & 5.96 & 5.76 & 1.00 & 1.00 & 0.00 & 0.0000 \\
\hline $40 \%$ & $40 \%$ & $8 \%$ & 0.25 & 6.22 & 6.02 & 1.00 & 1.00 & 0.00 & 0.0000 \\
\hline
\end{tabular}

Fuente: Bolsa de Valores de Lima y BCRP. 
Dado que se observa afectado el ROE, como se describe en el modelo (1), en la tabla 4, se muestra sensibilizada la prima de seguro (PUT) ante una posible variación del ROE, cuyos valores se observan en la columna PUT. Además, se visualiza, en términos tanto por uno, el precio de la prima del contrato que varía a medida que cambia el valor del ROE, mientras se mantienen constantes los otros factores, aplicado en el modelo PUT de Black \& Scholes. La prima del contrato (PUT) se ve encarecida en la medida que disminuye la rentabilidad patrimonial de las empresas. La figura 1 ilustra claramente el grado de protección que tendrían las empresas en la medida que hayan perdido valor en la rentabilidad patrimonial por efecto del FEN, cuya protección se potencia cuando el valor de subyacente toma valores por debajo de $14 \%$. La figura 2 ilustra la sensibilidad que tiene el precio del contrato ante la variabilidad del subyacente, en contraste a la potencia de la protección por parte del contrato. Se observa que se encarece la prima del contrato a medida que disminuye la rentabilidad patrimonial de las empresas.

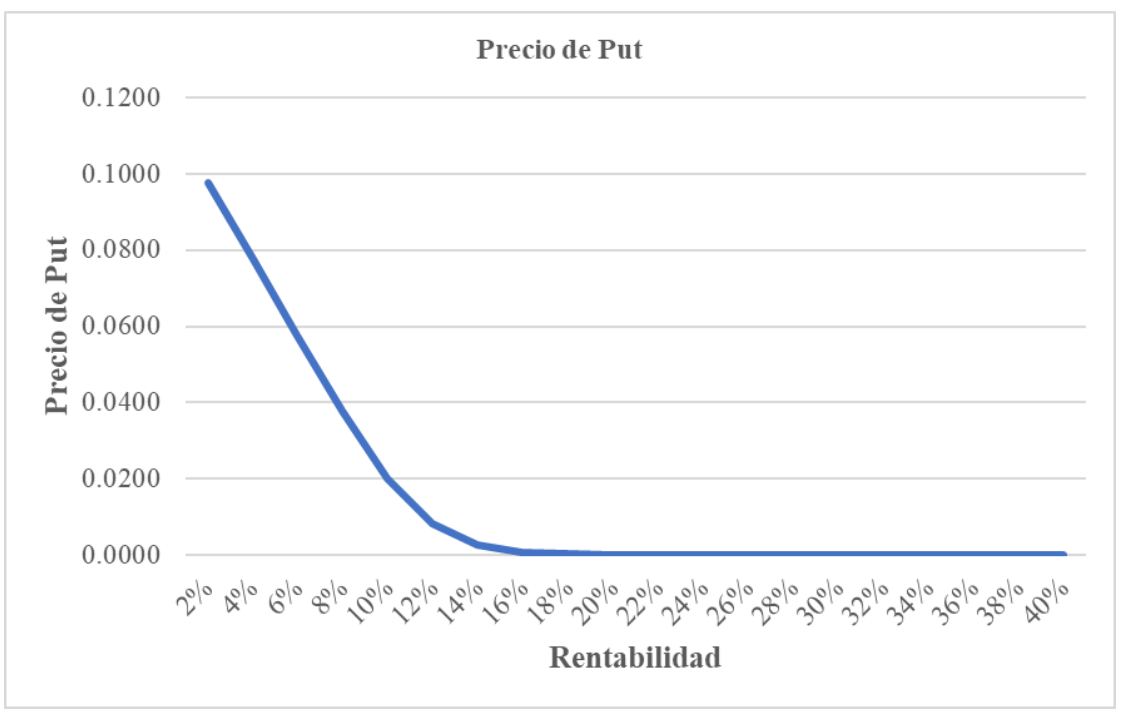

Figura 1: Precio del contrato PUT en posición larga.

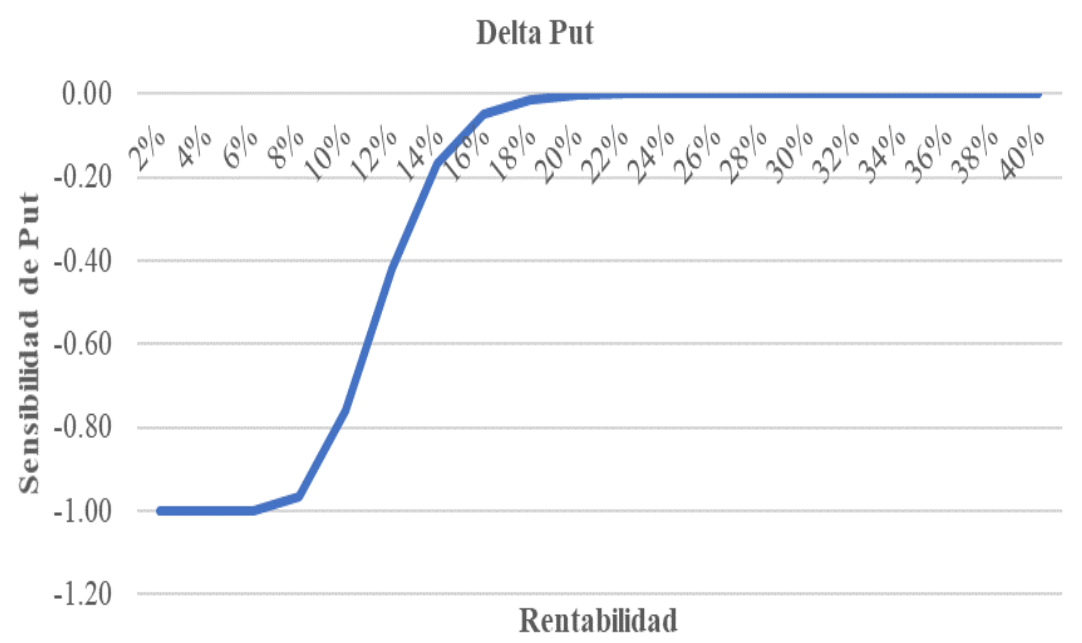

Figura 2: sensibilidad del precio del contrato PUT en posición larga.

\section{DISCUSIÓN}

En el modelo (1) se reflejan los efectos negativos ocasionados por el FEN y la temperatura sobre la rentabilidad patrimonial de las empresas pesqueras y agroindustriales peruanas, resultados que tienen relación con las conclusiones llegadas por Galarza y Kamiche (2012), que evidencia los efectivos negativos del FEN en la 
actividades económicas de pesca y agricultura del norte del Perú. Y por otro lado, Martelo (1998), en relación a las conclusiones a las que conducen los resultados del presente estudio, concuerdan en mostrar los efectos negativos causados por la variabilidad de la temperatura en la actividad de pesca y la agricultura.

En relación a la cobertura de riesgos con derivados, los estudios de Castro (2009) y Hull (2002) concluyen que la protección de riesgos climáticos con derivados financieros, que se negocian fuera del mercado organizado, otorgan importantes oportunidades de protección para las actividades de pesca y agricultura que enfrentan eventos de riesgos ante la presencia del FEN. Tal como se puede apreciar en los resultados mostrados a través del modelo (1) y los resultados mostrados en la tabla 3, asegurar con opciones PUT en posición larga para conservar el valor de la rentabilidad patrimonial de las empresas.

En el mismo sentido que describe Benito (2012) sobre los factores que influyen en el precio de la opción, en la investigación se determina que estos factores incidentes son el precio actual del subyacente, la rentabilidad patrimonial, el precio de ejercicio, la suma asegurada de la rentabilidad patrimonial esperada, el plazo del contrato, la volatilidad del subyacente así como la tasa libre de riesgo.

\section{CONCLUSIONES}

De los resultados presentados en la tabla 3 y modelo (1), se puede concluir que existen factores que influyen en la rentabilidad patrimonial de las empresas pesqueras y agroindustriales peruanas, como la variabilidad que ocurre con la temperatura, la presencia del FEN y lo que ocurre con la economía regional y sectorial. La disminución del ROE por efecto del FEN podrá ser asegurado con la suscripción del contrato OTC de opciones PUT en posición larga, tal como se aprecia en los valores presentados en la tabla 4 y las ilustraciones vertidas en la figura 1 y 2 , haciendo notar que el precio de los contratos se verán encarecidas cuando se presenta eventos de disminución del ROE por debajo del precio de ejercicio.

\section{REFERENCIAS}

Aguirre S. \& Méndez K. (2015). Incidencia del sector pesquero en el crecimiento económico en el Perú durante el periodo 1970-2014. Universidad Privada Antenor Orrego. Trujillo, Perú.

Banco Central de Reserva del Perú (BCRP). Recuperado de: http://www.bcrp.gob.pe/estadisticas.html

Benito, J. (2012). El Modelo de Black \& Scholes de Valoración de Opciones Financieras. Universidad de Barcelona. Barcelona, España.

Bolsa de Valores de Lima. Mercado al día. Recuperado de: https://www.bvl.com.pe/neg_rv_alfa.html

Caparrós, A. (2006). El informe Stern sobre la Economía del Cambio Climático. Mārid, España: Asociación española de ecología terrestre.

Castro, M. (2009). Managing Weather Risk with Rain Fall Option. Universidad Torcuato di Tella. Buenos Aires, Argentina.

Galarza, E. \& Kámiche, J. (2012). Impactos del Fenómeno de El Niño (FEN) en la economía regional de Piura, Lambayeque y La Libertad. Lima: GIZ.

Gutiérrez, M. \& Sueiros, J. (2015). Análisis sobre la transparencia en el Sector Pesquero Peruano. Programa de las Naciones Unidas.

Hull, J. (2002). Introducción a los Mercados de Futuros y Opciones. Madrid, España: Editorial Pearson.

Martelo, M. (1998). El Fenómeno del Niño. Ministerio del Ambiente y Recursos Naturales Renovables. Venezuela COMUNIICA, 3 (9) 48-55.

Ministerio del Ambiente MINAM (2016). El Perú y el cambio climático. Tercera comunicación Nacional del Perú a la convención Marco de las Naciones Unidad sobre el cambio climático. Hecho el depósito legal en la Biblioteca Nacional del Perú N²016-04430.

Olivares, B. \& Zingaretti, J. (2017). Análisis de la Sequía Meteorología en cuatro localidades agrícolas de Venezuela mediante la combinación de métodos multivariados. Universidad Nacional de Villa María, Córdoba, Argentina.

Soriano, Y. (2012). Análisis Comparativo de Métodos de Valuación de Opciones Asiáticas y con Barrera. México D.F.

Sociedad Nacional de Pesquería(2011). Memoria Anual de la Actividad Pesquera en el Perú. 